at whose suggestion the investigation was started and whose advice and assistance were of great help during its progress.

\title{
Summary
}

1. The densities and vapor pressures of ether have been measured over the lower temperature range.

2. From these and an equation of condition, the latent heats of evaporation and the change in internal energy on evaporation were calculated.

3. A relation connecting the specific volume of the liquid and the internal energy change on evaporation is given.

1. From the latent heats there was obtained the difference in saturation specific heats of the vapor and liquid, and from these, when compared with Regnault's values for the liquid, some values of the specific heat of the vapor at constant pressure were deduced.

Cambridge 39, MassachusetTs

[CONTRIBUTION From the Chemical Laboratory OF Clark University, I, 20]

\section{THE CONDUCTANCE OF SOLUTIONS OF SALTS IN PHENOL AT $50^{\circ}$}

\author{
By Charles A. Kraus and Herman F. Kurtz \\ Received August 2, 1922
}

Introduction.-The manner in which the conductance of an electrolyte in solution varies as a function of the concentration is largely governed by the dielectric constant of the solvent medium. In solvents of high dielectric constant, the equivalent conductance decreases continuously with increasing concentration up to very high concentrations. With solvents of intermediate dielectric constant-in the neighborhood of 20-, the equivalent conductance decreases with increasing concentration at low concentration and apparently approaches a more or less constant value in the neighborhood of normal concentration. With solvents of dielectric constant lower than 20 , the conductance decreases with increasing concentration at low concentration, and passes through a minimum at higher concentration, after which the conductance increases as the concentration increases. At concentrations considerably above normal, the conductance in all cases v1timately decreases with increasing concentration, presumably as a result of the influence of the increasing viscosity of the solutions. Finally, in solvents of very low dielectric constant the conductance increases with increasing concentration, beginning with the lowest concentrations at which reliable conductance measurements are available. This transformation of the form of the conductance curve as a consequence of the change in the value of the dielectric constant of the solvent medium is a very general phenomenon. $\mathrm{It}^{\circ}$ is strikingly illustrated by the conductance curves of a salt in a given sol- 
vent at different temperatures as, for example, in solutions of strong electrolytes in sulfur dioxide at temperatures between $-33.5^{\circ}$ and $+10^{\circ}$, as measured by Franklin. ${ }^{1}$ All these different forms of curves are remarkably well accounted for by the equation $(C \gamma)^{2} / C(1-\gamma)=D(C \gamma)^{m}+K$ as Kraus and Bray have shown. ${ }^{2}$ 'This equation, however, has been checked over a large concentration range only in the case of solutions in ammonia, sulfur dioxide and iso-amyl and -propyl alcohols. ${ }^{3}$ It is of interest to investigate the applicability of this equation to solvents of lower dielectric constant. The form of the curve is largely determined by the value of the exponent $m$ of the above equation. For values of $m$ less than unity, the conductance decreases with increasing concentration, while for values of $m$ greater than unity the conductance curve exhibits a minimum which is the more pronounced the greater the value of $m$. So far, no measurements are available on the conductance of dilute solutions over a large concentration range for solvents having a dielectric constant in the neighborhood of 10 . In order to supply data for a solvent of this type, conductance measurements have been carried out with solutions of tetramethylammonium iodide and of sodium iodide in phenol.

Apparatus, Materials and Procedure.-The conductance of the solutions was measured by the Kohlrausch method, observing the precautions now generally adopted in practice. A detailed description of the apparatus is unnecessary.

All measurements were carried out at $50^{\circ}$ in an oil thermostat whose temperature was maintained constant within $0.01^{\circ}$. The conductance cells were constructed of glass and were cylindrical in form, with permanent electrodes of the plate type. These cells were calibrated by comparison with a reference cell whose constant was determined by means of $0.1 \mathrm{~N}$ potassium chloride solutions.

The phenol used was purified by fractional distillation. The lowest value obtained for the specific resistance of the solvent was $0.146 \times 10^{-6}$. The solvent was collected directly in the cell in the course of distillation.

The tetramethylammonium iodide was an imported product and the sodium iodide was a domestic product of analyzed grade. Neither was further purified for the purpose of this investigation. In the calculations which follow, the density of phenol was assumed to be 1.058 , according to Hartung. ${ }^{4}$

The solutions were made up by introducing weighed amounts of salt into the cell in small glass buckets. Tetramethylammonium iodide dissolves fairly readily (in about an hour), but sodium iodide dissolves very slowly. It is for this reason that a larger number of measurements were not carried out with the latter salt.

Experimental Results.-In Table I are given the results of the conductance measurements with tetramethylammonium iodide and in Table II those with sodium iodide. In the tables, the concentration of the solution is given in the first column, the specific conductance of the solution corrected for the specific conductance of the solvent in the second column,

${ }^{1}$ Franklin, J. Phys. Chem., 15, 675 (1911).

${ }^{2}$ Kraus and Bray, Thrs Journat, 35, 1315 (1913).

${ }^{3}$ Keyes and Winninghoff, $i b i d$, , 38, 1178 (1916).

${ }^{4}$ Hartung, Z. physik. Chem., 77, 82 (1911). 
and the equivalent conductance of the electrolyte in the third column. In Table I, values of the equivalent conductance, calculated according to the preceding equation of Kraus and Bray, are given in the fourth column. At the head of each table is given $T$, the weight of phenol used; $L$, the specific conductance of the solvent; and $K$, the cell constant.

TABLE, I

Conductance of Tetramethylammonium Iodide in Phenol, at $50^{\circ}$

\begin{tabular}{|c|c|c|c|}
\hline \multicolumn{4}{|c|}{$W=307.88 \mathrm{~g} ., L=0.21551 \times 10^{-6}, K=0.01831$} \\
\hline$C \times 10^{3}$ & $L \times 10^{6}$ & $\Lambda_{\text {meas. }}$ & $\Lambda_{\text {calc }}$ \\
\hline 0.10769 & 1.3367 & 12.413 & 12.343 \\
\hline 0.22051 & 2.3025 & 10.441 & 10.436 \\
\hline 0.41026 & 3.5828 & 8.733 & 8.775 \\
\hline 0.81539 & 5.7018 & 6.093 & 7.047 \\
\hline 1.0120 & 8.9557 & 5.556 & 5.582 \\
\hline 3.2376 & 14.317 & 4.422 & 4.400 \\
\hline 6.4462 & 23.249 & 3.607 & 3.567 \\
\hline 12.790 & 39.319 & 3.074 & 3.050 \\
\hline 26.522 & 74,864 & 2.823 & 2.825 \\
\hline 35.532 & 100.26 & 2.822 & 2.830 \\
\hline 50.033 & 144.43 & 2.887 & 2.906 \\
\hline $7 \pm .547$ & 232.78 & 3.122 & 3.092 \\
\hline
\end{tabular}

'TABLE II

Conductance of Sodium Iodide in Phenor at $50^{\circ}$ $W=379.50 \mathrm{~g} ., \quad L=0.18047 \times 10^{-6}, K=0.01831$ $C \times 10^{3}+L \times 10^{6}, K=0.01831$

$\begin{array}{lll}0.113448 & 0.5539 & 4.883\end{array}$

$\begin{array}{lll}0.22875 & 0.9440 & 4.127\end{array}$

$\begin{array}{lll}0.41845 & 1.4338 & 3.426\end{array}$

$\begin{array}{lll}0.86481 & 2.2971 & 2.656\end{array}$

$\begin{array}{lll}1.6757 & 3.4994 & 2.088\end{array}$

Discussion.- The chief interest in this paper centers about the form of the conductance curve in a solvent of low dielectric constant. The dielectric constant of phenol, according to Drude, ${ }^{5}$ has a value of 9.58 at $48^{\circ}$. The experimental results were treated graphically according to the method of Kraus and Bray and the constants of the conductance function thus derived. The values found were: $\Lambda_{0}=16.67 ; D=0.70$; $m=1.28$; and $K=2.25 \times 10^{-4}$.

In the accompanying figure, the continuous curve represents the conductance function for the values of the constants given above. The experimental points are represented as crosses. As may be seen from the figure and from Col. 4 of Table $I$, the calculated and experimental values agree reasonably well. The maximum error is about $1 \%$ and the errors are well distributed. The agreement is as good as might be expected, considering the numerous sources of error affecting the measurement of the conductance of solutions in a solvent of this type at concentrations

s Drude, Z. pliyis. Chem, 23, 267 (1897). 
down to $10^{-4} \mathrm{~N}$. At the lower concentrations, the weight of salt is small and the errors due to weighing are, therefore, appreciable. Furthermore, the specific conductance of the solvent is relatively large, under which conditions the correction for the conductance due to the solvent is necessarily somewhat uncertain. Finally, the rate of solution of the salts is comparatively low, which necessitated frequent mixing of the contents of the cell, with a comparatively long interval of time elapsing between the measurements at the different concentrations.

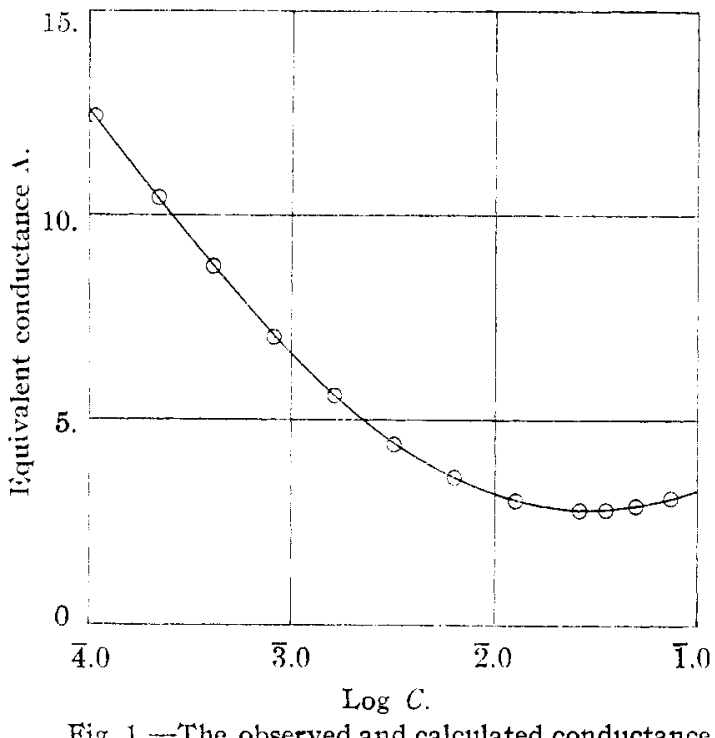

Fig. 1. - The observed and calculated conductance values of tetramethylammonium iodide in phenol.

The values of the constants are such as might be expected. The viscosity of phenol, as interpolated from the measurements of Scarpa, ${ }^{6}$ is 0.0342 . For the ratio of the equivalent conductance to the fluidity of the medium this gives a value of $0 . \tilde{n} 7$, which agrees well with that of other organic solvents. Kraus and Bray have shown that, for solvents having a dielectric constant below 25 , the value of the constant $D$ is very nearly independent of the nature of the solvent. The value found in the case of phenol is somewhat higher than that of most solvents for which data are available. As might be expected, the value of $K$ for tetramethylammonitum iodide is relatively low. Evidently, as the dielectric constant of the medium decreases, the value of this constant likewise decreases, as Kraus and Bray have previously pointed out. The value of 1.28 for the constant $m$ might have been anticipated from the value of the dielectric constant.

${ }^{6}$ Scarpa, Nuovo (imento, 5, 117 (1903); ibid., 6, 277 (1904); J. chim. phys., 2, 447 (1904). 
Very striking is the low value of the conductance of sodium iodide. If we assume that the limiting value of the equivalent conductance of sodium iodide is of the same order as that of tetramethylammonium iodide, the ionization of sodium iodide at a concentration of approximately $10^{-4}$ $N$ is only $25 \%$. As yet, we have little or no knowledge of the factors governing the ionization of different salts in a given solvent. This, however, is fairly clear: while the ionization of different binary salts in a solvent of high dielectric constant is very nearly the same for all salts, in solvents of low dielectric constant, the ionization varies greatly for different salts. In the case of phenol solutions, the difference in the behavior of sodium iodide and tetramethylammonium iodide illustrates the fact that in solvents of low dielectric constant the electrolyte, even at low concentration, possesses a much greater degree of individuality than it does in solvents of higher dielectric constant. It may also be noted, in this connection, that the conductance of a saturated potassium iodide solution, whose concentration is approximately $0.0045 \mathrm{~N}$, has a value of the order of 1.0.7 This is somewhat lower than might be expected from the data for a sodium iodide solution of the same concentration.

The freezing-point of a saturate dsolution of potassium iodide in phenol has been determined by Riesenfeld and found to be normal. The freezingpoint of various electrolytes, including tetramethylammonium iodide, has been measured by Hartung at concentrations as low as $0.01 \mathrm{~N}$. The values of $i$ found by Hartung are markedly greater than those corresponding to the ionization of the salt as determined by conductance measurements. In this connection, it may also be noted that Hartung obtained the greatest values of $i$ in the case of salts of weak acids. This indicates that hydrolysis occurs in these solutions as Hartung pointed out. To determine whether solutions of tetramethylammonium iodide are appreciably hydrolyzed in phenol, air was passed through a phenol solution of this salt at $50^{\circ}$, and thereafter through pure water for about an hour. The water gave no test for iodide either by the starch method or by silver nitrate. Evidently, the hydrolysis of tetramethylammonium iodide solutions is inappreciable and the discrepancy between the results of freezing-point and of conductance measurements cannot be accounted for in this way. Agreement between conductance and freezing-point measurements is not to be expected in the case of solutions in phenol, even at concentrations below $0.01 \mathrm{~N}$. If inter-ionic forces have an influence upon the properties of electrolytic systems, then it is to be expected that this influence will be the greater, the lower the dielectric constant of the medium, since the force due to a given charge under given conditions is the greater, the lower the value of the dielectric constant.

' R iesenfeld, Z. physik. Chem., 41, 346 (1902). 


\section{Summary}

1. The conductance of tetramethylammonium iodide in phenol at $50^{\circ}$ has been measured at concentrations from $1 \times 10^{-4} \mathrm{~N}$ to $0.07 \mathrm{~N}$. The constants of the conductance function found for this electrolyte are $\Lambda_{0}=16.67, D=0.70, m=1.28$, and $K=2.25 \times 10^{-4}$.

2. The conductance of sodium iodide solutions in phenol at the same temperature has been measured at concentrations from $1 \times 10^{-4}$ to $1.6 \times 10^{-3} \mathrm{~N}$. The ionization of sodium iodide is much lower than that of tetramethylammonium iodide.

3. The results are briefly discussed in relation to the freezing-point determinations of Hartung.

WORCESTER, MASSACHUSETTS

[Contributions from the Chemical, Laboratory of Clark University, I, 19]

\section{AN IMPROVED STILL FOR PRODUCING PURE WATER}

\section{By Charles A. Kraus and Wilbur B. Dexter}

Received August 3, 1922

Introduction.-One of the difficulties encountered in the study of the properties of dil. aqueous solutions is due to the lack of a convenient method for the preparation of pure water in larger quantities. While water having a specific conductance approaching that of pure water, $0.042 \times 10^{-6}$ at $18^{\circ}$, has previously been obtained by several investigators, a convenient method for obtaining such water in larger quantities and without undue preliminary preparation appears not to have been developed. The still described below has been found to meet general laboratory requirements admirably.

A detailed discussion of previous methods is omitted for the sake of economy of space. ${ }^{1}$ It may be noted, however, that the only methods which are practicable from a laboratory standpoint are those of Bourdillon and Weiland, who removed the chief impurity, which is carbon dioxide, by means of a stream of pure air passing through the condensing system during the process of distillation.

In the method of preparation described below, the use of pure air in the course of the distillation process has been eliminated. At the same time, the still has been so designed that it can be constructed by an ordinary tinsmith. Since the limit of the purity of the water obtained by the ordinary methods of distillation appears not to have been investigated systematically heretofore, a brief description may be given of some pre-

${ }^{1}$ Kohlrausch, Ann. Physik, 44, 577 (1891). Kohlrausch and Holborn, "Leitvermögen der Elektrolyte," Teubner, Leipzig, 1898, pp. 111-115. Kohlrausch and Heydweiller, Ann. Physik, 53, 209 (1894). Bourdillon, J. Chem. Soc., 103, 791 (1913). Weiland, This Journal, 40, 131 (1918). 